\title{
Evaluation of Fourier and wavelet analysis for efficient recognition of broken rotor bar in squirrel- cage induction machine
}

\begin{abstract}
Incipient fault detection of the induction machines (IM) prevents the unscheduled downtime and hence reduces maintenance costs. The Motor Current Signature Analysis (MCSA) is considered as an effective fault detection method in any IM. However, a signal processing technique, which enhances the fault signature and suppress the dominant system dynamics and noise must be considered. Frequency analysis as well as time-frequency analysis is the most common signal processing methods. In this paper, the effectiveness of these two analysis methods were investigated for incipient broken rotor bar detection. Wavelet transform provides more accurate failure detection in different operational circumstances. However, there are different families in the wavelet analysis that affect the efficiency of encoding, denoising, compressing, decomposing and reconstructing the signal under observation. Accordingly, it is desirable to select the powerful wavelet family, which produces the best results for the signal being analyzed. This research also investigated the analysis of current signal using different families of wavelet for effective detection of broken rotor bars in IM.
\end{abstract}

Keyword: Induction machine; Broken rotor bar; Motor current signature analysis; Fourier analysis; Wavelet analysis 